\title{
Democracia liberal en la teoría de la justicia según John Rawls
}

\author{
Liberal democracy in the theory of justice by John Rawls \\ EDGAR PALACIO MIZRAHI \\ Magister en desarrollo social, especialista en Estudios Político-Económicos, \\ en educación en Derechos Humanos, Doctorando en Ciencias Politicas. \\ Profesor de la Corporación Universitaria de la Costa, CUC. \\ edgarpalaciocamara@hotmail.com
}

Recibido: Marzo 2 de 2013

Aceptado: Mayo 25 de 2013

RESUMEN

Este artículo de reflexión analiza la Teoría de la Justicia de John Rawls, comos una nueva defensa de la democracia liberal para construir un orden social más justo, considera que la idea de justicia como equidad no tiene por qué limitarse a las relaciones entre los ciudadanos dentro de una comunidad política, sino que puede extenderse a las relaciones entre Estados y que una vez estos doten a sus ciudadanos de las necesidades básicas, estos empezaran a luchar por su libertad personal, que llevara así al estado ideal de justicia en todo Estado Social de Derecho.

Palabras Clave: John Rawls: Teoría de la Justicia, Estado Social de Derecho, Libertad, Estados, Ciudadanos, Política.

\begin{abstract}
The Theory of Justice by John Rawls is a new defense of liberal democracy to build a more just social order. It believes that the idea of justice as fairness does not have to be limited to relations between citizens within a political community, but it may be extended to relations between States. Once they provide their citizens with basic needs, these citizens began to fight for their personal freedom, which take well to the ideal state of justice in any rule of law.

Keywords: John Rawls, A Theory of Justice, Rule of Law, Freedom, States, Citizens, and Politics.
\end{abstract}

John Rawls filosofo político norteamericano del siglo XX (1921-2000), conocido por su teoría sobre la justicia, se convirtió en uno de los clásicos contemporáneos en la fundamentación liberal del Estado, en la década de los sesenta, cuando se inicio un debate muy serio sobre los fundamentos de la política y la validez de las institucio- nes demoliberales, los cuales se habían aceptado por mucho tiempo.

En este contexto surge la figura de John Rawls, profesor de Harvard, quien publico en 1972 uno de los libros más influyentes en el mundo académico: Teoría de la Justicia. 
Su trabajo es una nueva defensa de la democracia liberal. En su teoría podemos distinguir dos aspectos, uno es el método que sugiere y emplea y el otro las conclusiones a las que llega.

Su método sugiere que cuando al hombre le preocupan las cuestiones básicas de la justicia, o cuando desea descubrir las reglas que proporcionarían la estructura básica de una sociedad justa, se debe imaginar un congreso o asamblea constituyente de hombres y mujeres, que no pertenezcan a ninguna sociedad particular $y$, que se reúnan en una especie de convención constitucional con el fin de escoger las reglas fundamentales para una sociedad en formación.

Estas personas son comunes, del pueblo, tienen identidades, debilidades e intereses específicos. Pero sufren de un tipo de amnesia muy grave. No saben quiénes son, si son viejos o jóvenes, hombres o mujeres, blancos o negros, talentosos o tontos. En particular, y esto es muy importante, no conocen sus propias creencias acerca de que es valioso en la vida. Cada uno tiene su cosmovisión, es decir una concepción de cómo quiere que sea su propia vida. No tienen preferencias acerca de la moralidad sexual, pero no conocen cuáles son sus tesis acerca de esos temas, se encuentran separados de sus propias personalidades por un velo de ignorancia.

Ahora bien, estos amnésicos deben ponerse de acuerdo para determinar el contenido de una Constitución Política. A la pregunta, ¿cuál sería el acuerdo al que llegarían estas personas?, Rawls responde que debido a que ellas actúan solo en una búsqueda racional de su interés personal, llegan a principios de justicia.

Esto conlleva al segundo aspecto de su teoría ¿Qué conclusiones proporciona este método? Rawls las denomina "los dos principios de la justicia". El primero se refiere a ciertas libertades o derechos básicos que deben protegerse: libertades políticas convencionales, la libertad de votar, la libertad de expresión sobre los asuntos políticos y la libertad de conciencia y de religión, el derecho a la propiedad privada, el derecho a no ser detenido súbitamente y sin la debida causa, y el derecho a la protección personal, de esta forma quedan protegidas las denominadas libertades o derechos liberales convencionales.

El segundo es un principio más igualitario: se debe considerar la situación del grupo en peores condiciones. Todo cambio en la estructura social debe beneficiar a este grupo; supóngase que un cambio en la estructura económica hiciese mucho más rico al rico, mucho más pobre al pobre y a la clase media y, en general mucho más pobre a la sociedad en su conjunto, entonces el cambio no debe hacer- se, solo se puede hacer ese cambio, si el resultado ha de beneficiar al más pobre de los grupos por más pequeño que sea.

Estos dos principios están relacionados mediante lo que Rawls denomina (1995) "el principio de prioridad" (p.341). El primer principio domina al segundo, por ejemplo: No se deben restringir las libertades o derechos políticos, como el derecho a la libre expresión para beneficiar al grupo en peores condiciones de la sociedad, esto no se debe hacer. Solo cuando se ha protegido plenamente la libertad, se pueden considerar las cuestiones económicas que surgen del segundo principio.

Cuando se llega a estas consideraciones económicas se debe beneficiar a la clase en peores condiciones; pero esto no se puede hacer hasta que las libertades y derechos de todos estén suficientemente protegidas. En los párrafos siguientes se expondrá más ampliamente esta teoría de la justicia que puede ser muy útil en un país como Colombia, con miras a construir un orden social más justo, sin acabar con la democracia liberal, lanzándose a los brazos de cualquier tipo de totalitarismo, ya sea socialismo bolivariano a la criolla, o marxismo leninismo trasnochado, o cualquier tipo de fascismo.

El objetivo de Rawls (1971) al plantear su teoría de la justicia es combatir y superar el utilitarismo planteando que una teoría, por más elocuente que sea, debe ser rechazada o revisada si no es verdadera y que lo único que nos permite tolerar una teoría errónea es la falta de una mejor (p.17). Sin embargo, tampoco se plantea que su teoría es la más perfecta, sino que más bien se asume desde el comienzo mismo que se trata de una Teoría más y que de ninguna manera es la única que prevalece, o que esté por encima de las demás.

Además plantea que la idea principal del utilitarismo es que cuando las instituciones más importantes de la sociedad están dispuestas de tal modo que obtienen el mayor equilibrio neto de satisfacción distribuido entre todos los individuos pertenecientes a ella, entonces la sociedad está correctamente ordenada y es justa.

Para él es especialmente importante mostrar la superioridad de su teoría de justicia frente al utilitarismo. El principio de utilidad termina por identificar las nociones de lo bueno y de lo justo, al ver como justa la distribución de beneficios que maximice el bien, el cual el utilitarismo clásico asocia con la satisfacción del deseo. Así como un hombre, para realizar su propio bien, hace siempre un balance de pérdidas y ganancias de modo que en un momento pueda resultarle racional imponerse un sacrificio para obtener ganancias en el futuro, de la misma manera 
sería racional para una sociedad maximizar su bien, aun cuando en aras de lograr el mayor balance neto de satisfacción posible imponga sacrificios a una parte de sus miembros. Al hacer extensivo a la sociedad el principio utilitarista de elección individual, este principio se vuelve indiferente al modo de distribución de la suma de satisfacciones entre los individuos, lo que terminaría por justificar instituciones como la esclavitud, si los sacrificios de unos cuantos se vieran compensados ampliamente por la satisfacción de otros en el balance total.

En este sentido, no hay, en principio, razón por la cual las mayores ganancias de algunos no han de compensar las menores pérdidas de otros, o lo que es más importante, por qué la violación de las libertades de unos pocos no pudiera ser considerada correcta por un mayor bien compartido por muchos. Rawls plantea que, sin embargo, en un estado razonablemente avanzado de civilización, la suma mayor de ventajas no se alcanza de este modo ya que sin duda lo estricto de los aspectos de justicia del sentido común tiene cierta utilidad para limitar las propensiones humanas a la injusticia y a las acciones socialmente dañinas, aunque los utilitaristas crean que es un error afirmar esta severidad como un primer principio de la moral.

En el utilitarismo el bienestar social depende directa y únicamente de los niveles de satisfacción e insatisfacción de los individuos. Además, la satisfacción de los deseos tiene un valor por sí misma y necesariamente se toma en cuenta cuando se decide lo que es justo. De esta forma, al calcular el equilibrio mayor de satisfacción no importa sobre qué son los deseos, sino únicamente cómo su satisfacción afectaría el nivel de bienestar, primero de los individuos y luego de la sociedad como una suma de la satisfacción de los individuos.

Las críticas que se volvieron sobre el principio utilitarista de maximización de la utilidad reconocen tres núcleos temáticos. En primer lugar, se objeta la presunción de la comparabilidad interpersonal de la utilidad que subyace a la noción de suma de utilidades. En segunda instancia, esta suma de las utilidades no atiende a la forma en que el bienestar está distribuido en la sociedad, de modo que este criterio sería compatible con la coexistencia de pobreza y opulencia. Por último, se impugna la reducción del juicio moral a la utilidad, porque ésta, como experiencia subjetiva, puede adolecer de un desajuste con la realidad, o reflejar una concepción errónea acerca del bien, sea por falta de información o por creencias equivocadas.

La principal crítica que Rawls hace al utilitarismo es su falta de respeto por los individuos ya que en la versión más clásica, una persona no es considerada como valiosa y digna de protección por derecho propio. En lugar de ello es sólo una gota en el océano de la utilidad social general. Esto significa que algunas veces tendríamos que aceptar niveles muy bajos de utilidad para algunas personas si eso forma parte del esquema que maximiza la utilidad total (Elster, 1994).

Rawls plantea que, al contrario de lo que ocurre con el utilitarismo, las personas aceptan por anticipado un principio de igual libertad y lo hacen sin un conocimiento de sus fines más particulares y convienen en adecuar las concepciones de su bien a lo que requieren los principios de la justicia o, al menos, en no insistir en pretensiones que los violen directamente. En palabras de Rawls (1971) un individuo que se dé cuenta de que disfruta viendo a otras personas en una posición de menor libertad entiende que no tiene derechos de ninguna especie a este goce. El placer que obtiene de las privaciones de los demás es malo en sí mismo: es una satisfacción que exige la violación de un principio con el que estaría de acuerdo en la posición original (Rawls 1971, p. 41-42).

Así pues, Rawls construye una teoría alternativa que da respuesta al utilitarismo y, a la vez critica la falsedad detrás de los conceptos utilitaristas que no necesariamente resultan éticamente correctos y que mientras apelan al principio de mayorías, dejan fuera a muchos miembros de la sociedad (las minorías por ejemplo) lo cual es contraintuitivo en las democracias liberales modernas caracterizadas por el pluralismo.

Según John Rawls, toda sociedad humana se caracteriza tanto por la armonía como por el conflicto. Para que los hombres puedan crear una sociedad armoniosa y bien ordenada necesitan una serie de principios generales para determinar la parte justa que a cada uno le corresponde. Tales principios generales forman la base, el principio constituyente del orden social, y ofrece los criterios necesarios para evaluar sus instituciones, prácticas y políticas.

Para Rawls, ni Dios imparte los principios de justicia, como opinaron Agustín, Aquino y otros, ni se deducen de las leyes de la historia, de la estructura del universo ni de la razón inmutable del hombre, tal como argumentaron los historicistas, los estoicos, los teóricos de la ley natural y otros, sino que son producto de la deliberación y de la elección humana.

Por supuesto que la deliberación humana puede equivocarse y dar como resultado principios dudosos de justicia. En opinión de Rawls, aquel proceso será susceptible de generar principios ecuánimes de justicia en la medida en que se emprenda en condiciones «ideales», para él, tales 
condiciones existen cuando los hombres superan las diferencias que les separan, incluyendo desde los rasgos personales hasta las circunstancias naturales y sociales.

Rawls introduce así el concepto de "condición original», que significa una condición en la que han desaparecido o no existen características y circunstancias diferenciales entre los hombres, éstos son más o menos idénticos en su constitución y condición de vida, y no poseen nada que aun remotamente, pudiera formar la base de intereses personales mezquinos.

De esta manera están liberados de envidia, vanidad, codicia, afán del juego, capacidades naturales, rango social, color, religión, cultura, entorno familiar, posición de clase, conceptos específicos de excelencia, ambiciones, aspiraciones, proyectos para la vida, etcétera.

En resumen, se encuentran libres de todo lo que separa a un hombre de otros hombres de este tipo, constituidos y situados de manera más o menos idéntica, deliberan sobre los principios de la justicia y eligen aquellos que están dispuestos a aceptar como principios de obligado cumplimiento para ellos, siempre y bajo cualesquiera condiciones.

Todos se esfuerzan en promover sus intereses respectivos, y en "ganar para si mismo el índice más alto de bienes sociales primarios», sin tomar en cuenta qué lugar social ocuparán más adelante, ni qué capacidades y aptitudes llegarán a adquirir. Rawls está convencido de que intentarán protegerse contra las peores eventualidades, y elegirán los principios que les darán los acuerdos más ventajosos posibles, aun en el caso de que sus posiciones en la sociedad les fueran asignadas por sus enemigos.

Rawls (1995) está convencido que los hombres en la posición original, elegirían el siguiente concepto general de justicia:

...Todos los bienes sociales primarios -libertad y oportunidad, ingresos y riqueza, y los fundamentos de la dignidad personal- se distribuirán por igual, a menos que una distribución desigual de cualquiera de estos bienes o de todos ellos fuese ventajosa para los menos favorecidos

La concepción general implica dos principios de justicia, uno que trata de la distribución de la libertad y otro que se ocupa de la distribución de otros bienes primarios. Rawls (1995) formula el primer principio de la manera siguiente: Toda persona tendrá derecho por igual al más amplio sistema total de libertades básicas iguales compatible con un sistema similar de libertad para todos (p. 341).
El segundo principio se formula como sigue:

Se ordenarán las desigualdades sociales y económicas para que simultáneamente se logre: a) Ofrecer el mayor beneficio a los menos afortunados, dentro del principio del ahorro justo, y b) Que cargos y posiciones estén abiertos a todos en condiciones de una justa igualdad de oportunidad. Ya que pueden existir conflictos entre los dos principios, y entre las dos partes del segundo, Rawls indica las que él llama «reglas de prioridad»; con una sola excepción, estipulan que el primer principio tiene prioridad sobre el segundo, y que dentro de este último la segunda parte tiene prioridad sobre la primera.

Con referencia al primer principio, Rawls (1995) menciona cuatro «libertades básicas»: la política, que incluye el derecho de votar y de presentarse a cargos públicos, además de la libertad de expresión y de reunión; la libertad de conciencia, que incluye pensamiento y creencias; la libertad de la persona y derecho a la propiedad personal; y en cuarto lugar, la libertad frente al arresto arbitrario y rapto. Merece notarse que Rawls excluye deliberadamente de la lista de libertades básicas el derecho a ser propietario de los medios de producción y la libertad de contrato, tal como los liberales laissez-faire los entienden (p. 341).

El segundo principio de Rawls no tiene mayor complicación. No está en contra de las desigualdades sociales y económicas perse, sino solamente contra aquellas que son injustificables. En primer lugar, deben redundar en beneficio de los menos afortunados, por los cuales Rawls no entiende «literalmente el individuo en peor situación», sino más bien cualquier ciudadano cuyos ingresos y medios estén por debajo del promedio nacional.

Rawls introduce el principio «del ahorro justo» para estar seguro de que las perspectivas de los menos aventajados en el presente no mejoren a expensas de los ahorros y las inversiones necesarias para el crecimiento futuro. En segundo lugar, la desigualdad social se justifica si el cargo o posición al cual se vincula está abierto a todos. Para Rawls no es suficiente que los hombres sean formalmente libres de competir por cargos y posiciones: también deben disfrutar de una «justa» igualdad de oportunidad de adquirir la formación necesaria.

Las reglas de prioridad de Rawls tampoco presentan grandes problemas. Para él, el primer principio antecede al segundo y es moralmente superior a éste. No obstante, añade que dichas reglas sólo se aplican después de alcanzado un cierto nivel de desarrollo o "civilización», antes de ese nivel no es injusto distribuir de manera desigual ni exigir a los ciudadanos que renuncien a algunas de las 
libertades fundamentales, a cambio de conseguir ventajas sociales y económicas.

Rawls cree que, una vez que el nivel de bienestar social alcanza el punto en que todas las necesidades básicas están satisfechas, los hombres empiezan a considerar la libertad como el mayor bien, persiguen intereses espirituales y culturales, se preocupan menos de sus posiciones sociales relativas y de las posesiones materiales, y se preocupan más de elegir por sí mismos sus respectivos proyectos de vida. En ningún momento explica Rawls por qué tal preferencia es una cuestión de justicia.

De las dos partes del segundo principio, Rawls opina que la segunda tiene prioridad sobre la primera, esto quiere decir que no se puede sacrificar el principio de igualdad de oportunidad a cambio de ventajas potenciales para los hombres que están en peor situación.

Además sostiene que la violación de la justa igualdad de oportunidad sólo puede justificarse si se puede demostrar que, de no proceder así, las oportunidades para las partes de la comunidad menos favorecidas estarían aún más limitadas.

Rawls imagina que, después de adoptar los principios de justicia, quienes se encontrasen en la posición original procederían a través de tres etapas más. Después de seleccionar dichos principios, los actores en la posición original eligen una Constitución justa, que Rawls entiende como aquella que «satisface los principios de justicia y está mejor calculada para conducir a una legislación justa y efectiva», solamente el primero de los principios de justicia tiene aplicación en esta etapa.

Después de la etapa constitucional llega al estado legislativo, en el que el tema de consideración es la justicia de las leyes y la política. Es en esta etapa cuando el segundo principio de justicia es de una importancia capital. Requiere que la política social y económica intente maximizar las expectativas a largo plazo de las personas menos favorecidas, bajo condiciones de una justa igualdad de oportunidad y garantizado el mantenimiento de iguales libertades básicas. En la cuarta y última etapa, el tema de consideración es la aplicación de las leyes a casos particulares por los jueces y administradores.

En este momento se descorre el «velo de la ignorancia», tal como Rawls lo entiende, la secuencia de las cuatro etapas es un «mecanismo» para ir estrechando cada vez más el campo de aplicación de los principios de justicia e ir especificando los «puntos de vista» desd e los cuales se solventarán los problemas de la justicia. Dado que el interés primordial de este artículo se centra en el pensa- miento político de Rawl, se verá su idea de lo que es un sistema político justo.

Para Rawls, en un sistema político justo la autoridad legislativa corresponde a un cuerpo representativo elegido para un período limitado sobre la base del sufragio universal.

El principio de igual libertad requiere que cada miembro de la legislatura represente un número igual de votantes, y que sea la mayoría quien tome las decisiones legislativas. Rawls argumenta que la constitución puede limitar el campo de acción y la autoridad de la mayoría, o bien exigiendo una mayor pluralidad para ciertos tipos de medidas legislativas o bien mediante una ley de derechos con categoría de ley fundamental.

En ese caso, la libertad política se hace menos amplia pero también más segura. En un sistema político justo todos los ciudadanos deberían disfrutar de igual derecho de participación, y sus libertades políticas deberían tener igual valor. Para asegurar esto Rawls propone una serie de medidas.

Rawls opina que los debates en la asamblea legislativa deben estar informados por el deseo de aprobar la ley más justa en relación con un asunto específico. Los representantes deberían superar sus perjuicios y circunstancias sociales, debatiendo y votando «solamente» según lo que, en su opinión, dictan los principios de justicia.

El resultado de sus votaciones es una indicación fiable de lo que es más probable que esté en armonía con los principios de la justicia. La mayoría no necesariamente tiene razón, y un ciudadano puede estar en desacuerdo con una decisión. Sin embargo, una ley que resulta de las deliberaciones de legisladores racionales que a conciencia intentan seguir los principios de justicia tiene más probabilidades de ser correcta moralmente.

Como se ha visto, el segundo principio de justicia requiere que el gobierno asegure una justa y efectiva igualdad de oportunidades. Esto implica varias cosas.

El gobierno debe asegurar igual oportunidad de educación y cultura para las personas que tengan similares dotes y motivaciones, o mediante subsidios a escuelas particulares o con el establecimiento de un sistema de escuelas públicas. También debe promover la igualdad de oportunidades económicas y sociales mediante un control de la conducta de empresas y asociaciones privadas, impidiendo que se creen barreras monopolísticas al acceso a las ocupaciones más deseables. Además, deben garantizarse ingresos mínimos a través de subsidios fa- 
miliares y pagos especiales durante enfermedad y paro laboral, o a través de mecanismos como suplementos graduales para ingresos bajos.

Rawls argumenta, además, que cuando las desigualdades de riqueza exceden de un cierto límite peligran los principios de igual libertad e igualdad de oportunidad. En tal caso, los ricos pueden ejercer una influencia indebida sobre el carácter de los debates políticos y sobre el contenido de la legislación, y las libertades de aquellos con medios limitados pierden su valor.

Además, las oportunidades de educación y cultura ya no están abiertas por igual a los que tengan las mismas dotes, y los cargos y posiciones sociales ya no están abiertos a todos por igual. Por lo tanto. Rawls argumenta que es preciso corregir la distribución de la riqueza por medios tales como impuestos sobre herencias, donaciones y gastos suntuarios.

Según Rawls, los ciudadanos tienen el deber natural de cumplir leyes justas aprobadas bajo una constitución justa. Esto lo requieren tanto el principio del deber natural como el de la justicia. Si la estructura social básica es justa, aun las leyes injustas son de obligado cumplimiento, a menos que traspasen los límites tolerables de la injusticia. Ningún procedimiento constitucional, por perfecto que sea, puede garantizar que todas las leyes siempre serán justas.

La mayoría puede carecer del conocimiento y juicio correspondientes, o puede dejarse guiar por intereses propios, o puede carecer de un sentido perfecto de justicia. Tenemos un «deber natural de civismo», de no utilizar los fallos inevitables de un sistema social como pretexto fácil para negarnos a cumplir con sus leyes. No obstante, en una sociedad por lo demás justa, cuando una ley o política es de una injusticia flagrante o representa un abuso manifiesto de confianza y poder, caduca el deber general de tolerar las imperfecciones humanas, y el ciudadano tiene tanto el «deber» como el «derecho» de desobedecer.

Lo primero, porque tiene el deber natural de apoyar las instituciones justas y de oponerse a la injusticia. Y lo segundo, porque «el emplear el aparato coercitivo del Estado para mantener instituciones manifiestamente injustas es en sí mismo una forma de fuerza ilegitima que finalmente los hombres tienen el derecho de resistir».

Para Rawls, la desobediencia del ciudadano debe, en primer lugar, tomar la forma de la desobediencia civil, que es un acto público, no violento, consciente y político; la violación deliberada de una ley específica para lograr un cambio deseado en una política o ley específica del go- bierno, es un acto público en el sentido de que se realiza en público y es un llamamiento al sentido público de la justicia; es civil porque mientras desobedece la ley el individuo reconoce plenamente la autoridad del gobierno y acepta las consecuencias legales de su acción; y es político en el sentido de que se dirige a la mayoría y se justifica no en términos de una moralidad religiosa o personal sino de un concepto de la justicia generalmente compartido y que subyace al orden político.

Según Rawls, la desobediencia civil es un «modo de dirigirse» al sentido de justicia de la mayoría, y es una declaración de que, en opinión de los participantes, los principios de la colaboración social no se están respetando.

Rawls argumenta que a veces la desobediencia civil puede no ser suficiente, y puede ser justificado que un ciudadano recurra a la acción militante y a la resistencia. Es claro que el ciudadano es libre de recurrir a ellas contra una estructura social o Constitución injusta. Como se ha notado, el individuo no tiene ningún deber natural de mantener las instituciones injustas, y si de combatirlas.

Rawls argumenta que, aun en una sociedad justa o casi justa, a veces puede ocurrir que la desobediencia civil no sea suficiente. Si la mayoría se comportara de una manera "gratuitamente injusta y abiertamente hostil» en sus relaciones con otros grupos, o si la desobediencia civil no lograse su objetivo de corregir las violaciones de los dos principios de justicia, y especialmente del primero, entonces podría hacerse necesaria la resistencia por la fuerza.

Rawls considera que la idea de justicia como equidad no tiene por qué limitarse a las relaciones entre los ciudadanos dentro de una comunidad política, sino que puede extenderse a las relaciones entre Estados. Podríamos definir la posición original de manera que incluyese no solamente a personas particulares sino también a representantes de diversas naciones que trabajasen tras el "velo de la ignorancia», puestos de acuerdo sobre unos principios obligatorios de justicia internacional que fuesen universales y eternos.

Rawls piensa que tales principios incluirían la igualdad de las naciones, el derecho de autodeterminación nacional, el derecho de autodefensa contra todo ataque externo, el deber de cumplir los tratados, la prohibición de ciertas formas de violencia en la guerra, una definición estricta y limitada del interés nacional y la exclusión de las ganancias económicas, la expansión territorial y la gloria nacional como razones justificables para la guerra.

Estos y otros principios públicos de justicia internacional no solamente regularían las relaciones entre los Estados, 
sino que también suministrarían los criterios por los cuales los ciudadanos pueden juzgar los actos de sus gobiernos. Un ciudadano podría apelar a ellos para justificar su negación en conciencia a tomar parte en una guerra injusta. Un soldado podría negarse a realizar ciertos actos si él creyera "de forma razonable y en conciencia» que viola los principios que gobiernan la conducción de la guerra.

Y podría negarse totalmente a tomar parte en una guerra si pudiera demostrar que viola los principios de la justicia internacional, y que, tomando todo en consideración, «su deber natural de no convertirse en agente de una grave injusticia y hacer mal a otro» pesara más que su deber de obedecer a su gobierno.

Ahora usaremos la teoría de la justicia de Rawls para tratar de aplicarla en la creación de un orden social justo, bajo el principio de: Una vez socializada la capacidad a favor de la necesidad, el mérito debe reinar.

¿Somos todos iguales? La modernidad occidental elevo los valores de libertad, propiedad e igualdad a derechos y lo hizo de tal manera que nos parece que ellos son conceptos claros, complementarios unos con otros y que apuntan al mismo desarrollo armónico del individuo y la sociedad. Lo primero que debemos observar es que ellos ni son tan claros ni tan complementarios y que no tienen el mismo estatuto con relación al individuo y su articulación.

La igualdad parece ser el más vinculante de los tres valores y esta tan cercano al valor de la justicia que casi se usa para definirla, como cuando se dice que justicia es tratar a todos por igual, base de lo que luego hemos llamado equidad, definida, en principio, como un tratar igual a los iguales y desigual a los desiguales. Lo que la equidad dice es que solo cierto criterio moral puede justificar un trato desigual de quienes deben ser, en principio, tomados como iguales.

La igualdad no tuvo siempre, ni tiene en todas las culturas distintas a la occidental, el mismo énfasis que en los últimos siglos ha venido a tener en occidente. Para solo reducirnos a occidente, baste con observar que ni entre los griegos, ni entre los romanos, ni entre los judíos todos los seres humanos eran considerados iguales. Los griegos, en general, no se consideraban iguales a los barbaros, ni entre ellos, consideraban que todos eran iguales, afirmaron como un hecho natural la desigualdad de los seres humanos, unos nacen para mandar y otros para obedecer, como lo plantea el filósofo griego del siglo IV a.c Aristóteles, en su obra la política. Los judíos se diferenciaron de los gentiles, pero es en el cristianismo donde comienza a gestarse la idea de que todos los seres humanos son iguales, dice el apóstol Pablo en Gálatas 3:28 "ya no hay judío ni griego; no hay esclavo ni libre; no hay varón ni mujer; porque todos vosotros sois uno en Cristo" Cristo deshace las diferencias religiosas, sociales y sexuales, todos somos iguales ante Dios; los varones judíos saludaban el nuevo día con la oración: "Señor, te doy gracias porque que no soy un gentil, un esclavo o una mujer", pero es en el cristianismo donde se afirma la igualdad esencial y jurídica de los seres humanos.

La Biblia no ignora las diferencias que caracterizan a las personas en diferentes aspectos. La diversidad racial o étnica, cultural, social, moral, política, etc. son un hecho, es algo dado. Pero tales diferencias son solo formales, secundarias, no esenciales, son accidentales. Lo importante para la Biblia es la igualdad esencial u Ontológica, trascendental y profunda de la familia humana. Cuando Job, por ejemplo, reflexiona respecto de los derechos de sus esclavos, para la biblia los esclavos tenían derechos, se pregunta: "si hubiera tenido en poco el derecho de mi esclavo y de mi esclava, cuando ellos contendían conmigo, ¿qué haría yo cuando Dios se levantase? y cuando el preguntara, ¿qué le respondería yo? El que en el vientre me hizo a mí, ¿no lo hizo a el? ¿Y no nos dispuso uno mismo en la matriz? Job 31:13-15.

Durante la edad media tampoco se consideró jurídica y políticamente a todos los seres humanos como iguales. Esto solo vino a ser posible en la modernidad. La modernidad trajo la igualdad primero como un ideal y después impuso el hecho normativo de la igualdad y lo hizo a través tanto de discursos filosóficos como políticos que invocaban la igualdad tanto en términos descriptivos como normativos. Una cosa es decir, que todos los seres humanos son iguales, en un sentido primero fisiológico y después mental, como una descripción objetiva del mundo de las cosas, y otra muy distinta, al menos en principio, decir que todos los seres humanos deben ser tratados como iguales, es decir, como si fueran iguales aunque no lo sean. La primera oración enuncia un hecho y la segunda una norma que luego pudo convertirse, a través de la retórica de los derechos del hombre, en un derecho. La primera oración compara a los seres humanos y no encuentra diferencias significativas. La segunda dice que no debemos mirar las diferencias porque ellas no son moral o jurídicamente, significativas. En el lenguaje cristiano, ese algo era el ser hijos de Dios y tener un alma inmortal. El lenguaje moderno transformo esto en algo como tener una esencia para la libertad, o una dignidad, o ser una persona de derecho.

Desde la República de Platón, donde la indagación por el régimen político ideal comienza como una indaga- 
ción por la justicia, ha sido una constante en occidente el vincular la justicia a la reflexión filosófica sobre el orden social. Hay preguntas más o menos constantes: ¿Hay justicia en la forma como la naturaleza, las leyes del mercado o las estructuras sociales asignan a los individuos las cargas y los beneficios? ¿Debemos intervenir esos métodos de asignación y redistribuir las cosas de otro modo? Si la respuesta es sí ¿cómo y por qué se debe redistribuir? Y, sobre todo, ¿que está disponible para la redistribución? ¿Cuál ha de ser el criterio moral que debe presidir esa redistribución? ¿Debe ese criterio aplicarse preferencialmente a las instancias sociales que afectan las expectativas de los seres humanos o solo a cierta institucionalidad política? Todas estas preguntas se pueden resumir en una sola: ¿cuál es el orden social justo?

La noción de equidad, que es analíticamente distinguible de la de justicia pero que tiende a ser ligada a ella, ha sido definida de modos distintos desde Aristóteles, quien pasa por ser el primero en haberla incorporado a la llamada regla de justicia.

La justicia aparece de alguna forma ligada a la igualdad antes que a la libertad, la felicidad, la bondad, etc. Casi se podría definir la justicia diciendo que consiste en tratar a todos por igual. Por eso la justicia es representada como aquella virtud que se cubre los ojos con una venda a fin de permanecer ciega ante las diferencias Pero, como se sabe, esta igualdad puede ser una gran injusticia. El filósofo griego Aristóteles, en el libro V de su Ética Nicomaquea, reflexiono que tratar a todos por igual, eso que también llego a conocerse como igualdad aritmética, comportaba una indiferencia ante ciertos criterios que debía tomar en cuenta quien se proponía ser justo. Corrigió entonces esta igualdad aritmética con otra que, por oposición con la anterior, podría llamarse igualdad geométrica o igualdad pitagórica y que define la regla de la justicia como tratar igual a los iguales y desigual a los desiguales. Pero ¿igualdad y desigualdad con referencia a qué? la equidad que es otro nombre para esa igualdad geométrica, requiere entonces un criterio adicional.

Para definir ese criterio, la filosofía occidental ha oscilado entre tres valores: merito, necesidad y capacidad. Se hará uso de un ejemplo para ilustrar las diferencias entre ellos. Un alcalde decide financiar diez becas para estudios universitarios. Esas becas hay que repartirlas justamente. ¿Pero justamente en qué sentido? Otorgándole las becas a los diez mejores bachilleres de su municipio. Basta con tomar los puntajes del icfes y escoger los diez más altos.se premia el mérito de haber logrado tales puntajes. Así, pues, habrá obrado con justicia. Pero, objetaran algunos, entre los puntajes más altos están los que tienen más conocimientos, que no son necesaria- mente los que tienen mejores capacidades. Un estudiante pudo haber repetido los años, estudiado el doble que sus compañeros, pudo haber pagado profesores extras que otros no podrían proveerse, pudo haberse matriculado en preicfes, etc., de forma tal que alcanzo altos puntajes, sin ser necesariamente un estudiante con las mejores capacidades. El alcalde puede defenderse diciendo que él no está becando las capacidades sino el mérito o los logros o puede optar por becar sencillamente los mejores de acuerdo a sus capacidades, tengan o no los mejores puntajes en conocimiento. Así que tendrá que realizar unas nuevas pruebas que midan las aptitudes, las facilidades o habilidades potenciales del estudiante para resolver cierto tipo de problemas. No premiara a los que han tenido mejores oportunidades, mejores escuelas por tener más recursos, sino a quienes prometan mas así no hayan tenido oportunidad de desarrollar sus talentos. Cualquiera de las dos decisiones contempladas hasta ahora puede ser cuestionada desde un tercer punto de vista. No se trataría de becar a los que tienen más conocimientos o a los que tienen más capacidades, sino a los que tienen más necesidades o los menos favorecidos socialmente. ¿Por qué becar a quienes pueden sufragar o pagar sus estudios por sí mismo? los dineros públicos cumplen su función cuando atienden a las necesidades. Por consiguiente, no hay que mirar el icfes sino las declaraciones de renta, la situación económica de las familias de los aspirantes. ¿Qué debe, hacer en ultimas el alcalde? Similares problemas se pueden presentar con referencia a una decisión en materia de vivienda, pavimentación de calles, cobro de servicios públicos, valorización, inversión en educación pública, etc.

Si bien es justo que cada cual goce lo que logra por su propio esfuerzo, no todos están en las mismas condiciones para lograr sus fines, porque unos hacen incluso más esfuerzo que otros y no alcanzan los mismos fines. Se requiere entonces igualar los puntos de partida, a fin de permitir, ahora si realmente, que los que perseveran más y llegan más lejos tengan un mejor nivel de vida como un premio a su esfuerzo.

\section{Conclusiones}

Se puede resumir las tesis presentadas por John Rawls en su libro, teoría de la justicia, diciendo que presenta dos aspectos fundamentales en su obra. Primero el método que sugiere y emplea, y segundo las conclusiones a las que llega. Este método es llamativo pues sugiere que cuando al hombre le preocupan las cuestiones básicas de la justicia o cuando desea descubrir las reglas que proporcionarían la estructura básica de una sociedad justa, debemos imaginar un congreso de hombres y mujeres, que aún no pertenezcan a ninguna sociedad particular y que 
se hayan reunido en una especie de convención institucional con el fin de escoger las reglas fundamentales para una sociedad en formación.

A la pregunta, cuál sería el acuerdo al que llegarían estas personas, Rawls responde que debido a que ellas actúan solo en una búsqueda racional de su interés personal, llegan a principios de justicia. Esto nos lleva al segundo aspecto de su libro, ¿qué conclusiones proporciona este método?, Rawls las denomina los dos principios de la justicia. El primero habla de ciertas libertades básicas que deben protegerse y el segundo es un principio más igualitario: Debemos considerar la situación del grupo en peores condiciones. Todo cambio en la estructura social ha de beneficiar a ese grupo. Finalmente, los dos principios están relacionados mediante lo que Rawls denomina, el principio de prioridad, el primer principio domina al segundo.

\section{Referencias}

Arendt, H, (1998) Los orígenes del totalitarismo, taurus, Madrid.

Barry, B, (1989) Theories of Justice, University of California Press, Berkeley.

Botero, J (ed.), (2005) Con Rawls y contra Rawls. Una aproximación a la filosofía política contemporánea, Universidad Nacional de Colombia-Unibiblos, Bogotá.

Cortes, F (1999). De la política de la libertad a la política de la igualdad, Siglo del Hombre Editores y Universidad de Antioquia, Bogotá.

Elster, J (1994). Justicia local, Gedisa, España.

Fried, Ch (1988), ¿Es posible la libertad? En libertad, igualdad y derecho, editado por Sterling M.McMurrin, Ariel Derecho, Barcelona.

Grueso, I (Comp.), (2005) John Rawls: legado de un pensamiento, Editorial Universidad del Valle, Cali.

Grueso, I, (2003) La metafilosofía rawlsiana, en Phronesis, No 10, 2003.

Grueso, I (2000) La Justicia como equidad como una teoría moral Kantiana, en Humanidades, UIS.

Grueso, I, (1977). Rawls: una hermenéutica pragmática, Editorial Universidad del valle, Cali.

Habermas, J, (1998) Between Facts and Norms, The MIT Press, Cambridge, MA.

Habermas, J, (1998) Reconciliación mediante el uso público de la razon, en Jurgen Habermas, j y Rawls, J Debate sobre el liberalismo político, Paidos, Barcelona.

Kant, E (1980) Fundamentación de la metafísica de las costumbres, Espasa-Calpe, Madrid.

Nozick, R, (1988) Anarquía, Estado y Utopía, FCE, México.

Popper, K, (1981) La sociedad abierta y sus enemigos, Paidos, Barcelona.

Rawls, J (1995) A Theory of Justice, Harvard University Press, Cambridge, Mass, 1971.version castellana: Teoría de la Justicia, FCE, México.

Rawls, J, (1971) Teoría de la Justicia, FCE, México.

Rawls, J, (1999) Justicia como equidad, tecnos, Madrid.

Sen, A. (2000), Desarrollo y libertad, planeta, Barcelona.

Van Parijs, P (1996) Libertad real para todos, Paidos, Barcelona.

Van Parijs, P (1993) ¿Que es una sociedad Justa?, Editorial Ariel, Barcelona. 